\title{
Gravitational sedimentation of flocculated waste activated sludge
}

\author{
C.P. Chu ${ }^{\mathrm{a}}$, D.J. Lee ${ }^{\mathrm{a}, *}$, J.H. Tay ${ }^{\mathrm{b}}$ \\ a Department of Chemical Engineering, National Taiwan University, Taipei 10617, Taiwan \\ ${ }^{\mathrm{b}}$ Division of Environmental and Water Resources Engineering, School of Civil and Environmental Engineering, \\ Nanyang Technological University, Nanyang Ave., Singapore 639798, Singapore
}

Received 31 August 2001; received in revised form 28 May 2002; accepted 3 June 2002

\begin{abstract}
The sedimentation characteristics of flocculated wastewater sludge have not been satisfactorily explored using the non-destructive techniques, partially owing to the rather low solid content (ca. 1-2\%) commonly noted in the biological sediments. This paper investigated, for the first time, the spatial-temporal gravitational settling characteristics of original and polyelectrolyte flocculated waste activated sludge using Computerized Axial Tomography Scanner. The waste activated sludge possessed a distinct settling characteristic from the kaolin slurries. The waste activated sludges settled more slowly and reached a lower solid fraction in the final sediment than the latter. Flocculation markedly enhanced the settleability of both sludges. Although the maximum achievable solid contents for the kaolin slurries were reduced, flocculation had little effects on the activated sludge. The purely plastic rheological model by Buscall and White (J Chem Soc Faraday Trans 1(83) (1987) 873) interpreted the consolidating sediment data, while the purely elastic model by Tiller and Leu (J. Chin. Inst. Chem. Eng. 11 (1980) 61) described the final equilibrated sediment. Flocculation produced lower yield stress during transient settling, thereby resulting in the more easily consolidated sludge than the original sample. Meanwhile, the flocculated activated sludge was stiffer in the final sediment than in the original sample. The data reported herein are valuable to the theories development for clarifier design and operation. (C) 2002 Elsevier Science Ltd. All rights reserved.
\end{abstract}

Keywords: Activated sludge; CATSCAN; Flocculation; Elastic; Plastic; Constitutive equation

\section{Introduction}

Slurry sedimentation characteristics are essential to the design of thickener in water and wastewater industries. Traditional flux theory ignored the role of the bottom sediment [1-3]. Dixon [4] discussed the compression of sediment that traditional flux theory does not account for. Tiller [5] and Fitch [6] refined the Kynch theory to consider the effect of increasing sediment during sedimentation. Font $[7,8]$ reviewed the works considering the compression zone effects in batch sedimentation.

\footnotetext{
*Corresponding author. Fax: + 886-2-2362-3040.

E-mail address: djlee@ccms.ntu.edu.tw (D.J. Lee).
}

The way the solid fraction (volume fraction of solid phase, $\phi)$ changes with solid pressure $\left(P_{\mathrm{s}}\right)$ controls the rheological characteristics of the consolidating sediment. As Koenders and Wakeman [9] revealed, most related works assumed an elastic filter cake having the following power-law type constitutive equation:

$\phi=\phi_{\mathrm{g}}\left(a+\frac{P_{\mathrm{s}}}{P_{0}}\right)^{\beta}$

where $a$ can be taken as 0 or $1 . \phi_{\mathrm{g}}$ is the null-stress solid fraction or the gel point if $a=1$ [37]. $P_{0}$ represents the characteristic solid pressure [10]. $\beta$ denotes the compressibility of the sediment subjected to external stress. Eq. (1) assumes that $\phi$ depends sorely on the local solid pressure $P_{\mathrm{s}}$. Hence the solid fraction in a consolidating 


\begin{tabular}{|c|c|c|}
\hline \multicolumn{2}{|c|}{ Nomenclature } & \multirow{3}{*}{$\begin{array}{ll}z & \text { vertical distance from the coordinate position- } \\
\text { ing downward with its origin located at the top } \\
\text { of sediment }(\mathrm{m})\end{array}$} \\
\hline$a$ & parameter in Eq. (1) (dimensionless) & \\
\hline & gravitational acceleration $\left(\mathrm{m} / \mathrm{s}^{2}\right)$ & \\
\hline$K$ & hindered coefficient (dimensionless) & Greek letters \\
\hline$\kappa$ & dynamic compressibility in Eq. (2) $(\mathrm{m} \mathrm{s} / \mathrm{kg})$ & empirical constant in Eq. (1) (dimensionless) \\
\hline$L_{\mathrm{c}}$ & height of sediment starts to yield (m) & dynamic compressibility in Eq. (2) $(\mathrm{m} \mathrm{s} / \mathrm{kg})$ \\
\hline$P_{0}$ & characteristic pressure in Eq. (1) $(\mathrm{Pa})$ & solid fraction (dimensionless) \\
\hline$P_{\mathrm{s}}$ & solid pressure $(\mathrm{Pa})$ & null-stress solid fraction (dimensionless) \\
\hline$P_{\mathrm{y}}$ & yield stress $(\mathrm{Pa})$ & initial solid concentration of clay slurry \\
\hline$t$ & settling time (s) & (dimensionless) \\
\hline$U_{0}$ & Stokes velocity $(\mathrm{m} / \mathrm{s})$ & density difference between solid and liquid \\
\hline$u_{\mathrm{S}}$ & solid velocity $(\mathrm{m} / \mathrm{s})$ & $\left(\mathrm{kg} / \mathrm{m}^{3}\right)$ \\
\hline$y$ & the height of supernatant-slurry interface $(\mathrm{m})$ & \\
\hline
\end{tabular}

sediment would vary continuously along the vertical axis from the settler bottom.

Buscall and White [11] proposed a consolidation model considering the flocculated slurry as a purely plastic body that possesses a yield stress $\left(P_{\mathrm{y}}(\phi)\right)$. If the local pressure of sediment exceeds $P_{\mathrm{y}}$, the network structure would yield. Buscall and White [11] proposed the following constitutive equation:

$\frac{\mathrm{D} \phi}{\mathrm{D} t}=\kappa(\phi, P)\left[P-P_{\mathrm{y}}(\phi)\right]$,

if $P>P_{\mathrm{y}}(\phi)$. Otherwise, $\mathrm{D} \phi / \mathrm{D} t=0$, that is, no solid consolidation would occur in the sediment. $\kappa$ is called the "dynamic compressibility" of the suspension. This model had been extensively applied to sedimentation processes of flocculated suspensions (see, for instance Refs. [12-15]). If the sediment were purely plastic, there should exist a constant solid fraction portion in the sediment in which the solid stress has not exceeded the yield stress.

Researchers have adopted several non-destructive techniques to obtain the relevant variable in the solid/ liquid separation processes as a function of space and time, such as $\gamma$-ray [13,16], NMR/MRI [17-19], and Xray [12,20-26]. Buscall [27] reviewed the non-destructive techniques in solid-fluid system.

Polyelectrolyte conditioning has long been utilized to pre-treat the sludge in order to increase its filterability [28]. However, few works in literature investigated the sedimentation characteristics of flocculated wastewater sludge using the above-mentioned, non-destructive techniques, partially owing to the rather low solid content (ca. 1-2\%) commonly noted in the biological sediments. We built up the solid fraction distributions in this paper for the original and flocculated waste activated sludge with repeated scanning using a Computerized Axial Tomography Scanner (CATSCAN). A kaolin slurry was used as the reference system for comparison.

\section{Experimental}

\subsection{Sample}

Waste activated sludge sample was collected from the 69th Street Wastewater Treatment Plant, Houston, TX on December 14, 1998. The pH of the sludge was 6.5. The weight fraction was $1.3 \%(\mathrm{w} / \mathrm{w})$. An Accupyc Pycometer 1330 (Micromeritics) measured the solid density as $1630 \mathrm{~kg} / \mathrm{m}^{3}$. Therefore the initial solid fraction of the sludge was around $0.8 \%(\mathrm{v} / \mathrm{v})$.

Kaolin slurry was prepared by mixing prescribed amount of powders with $0.1 \mathrm{M} \mathrm{NaClO}_{4}$ solution at a solid fraction of $1 \%(\mathrm{v} / \mathrm{v})$. The addition of $\mathrm{NaClO}_{4}$ provided a high ionic strength to prevent the interference of any ions that might be released from the particle surfaces. The $\mathrm{pH}$ value was fixed at 7.0. An Accupyc Pycnometer 1330 (Micromeritics) measured the solid density as $2730 \mathrm{~kg} / \mathrm{m}^{3}$.

A cationic polyelectrolyte denoted as polymer T-3052 was obtained from Kai-Guan Inc., Taiwan. The polymer T-3052 is a polyacrylamide with an average molecular weight of $10^{7}$ and a charge density of $20 \%$. The polymer solution was gradually poured into the original slurry with $200 \mathrm{rpm}$ of stirring for $5 \mathrm{~min}$, followed by $50 \mathrm{rpm}$ for another $20 \mathrm{~min}$. A small quantity of floc-polymer aggregates was transferred carefully into the fresh electrolyte at the same $\mathrm{pH}$ and electrolyte concentration as the original electrolyte after mixing and prior to settling. The $\zeta$ potentials of the floc-polymer aggregates were measured by the Zetameter (Zeter-Meter System 3.0, Zeter-Meter Inc., USA). Table 1 lists the surface charge and the floc size data. For both sludges, the floc size was increased monotonically with the flocculant dose, and the $\zeta$ potential neutralized at $0.8 \mathrm{~g} / \mathrm{kg}$ dried solid (DS) for kaolin slurry and $15 \mathrm{~g} / \mathrm{kg}$ DS for the waste activated sludge, respectively. 


\subsection{CATSCAN}

Batch settling experiments were conducted in a settling cylinder that had a height of $20 \mathrm{~cm}$ and a diameter of $9.5 \mathrm{~cm}$, and were continuously monitored with the CATSCAN (DeltaScan 2060; $100 \mathrm{kV}, 75 \mathrm{~mA}$ ). The X-ray attenuation, which is normally presented as the CT number [29], could be obtained from a horizontal section of settling sludge at a thickness of $0.5 \mathrm{~mm}$. Bergstrom [13] adopted similar procedures using their $\gamma$-ray apparatus. Preliminary tests revealed that the CT number for the solution that contained flocculant (no solid particles) was essentially zero since the organic compounds did not absorb a significant amount of X-ray. Furthermore, the CT-number was found to increase monotonically with the solid fraction in the sludge (Fig. 1). The uncertainties in axial position measurement and the local solid volume fraction were $2.5 \mathrm{~mm}$ and $0.1 \%$, respectively, close to those reported in Auzerais et al. [12].

Fig. 2 demonstrates some cross-sectional CATSCAN images for the kaolin and the biological sludge sediments. The luminescence intensity $(0-255$ in gray scale) increased according to the solids fraction, with dark portions in Fig. 2 denoting the pore and the bright ones the solids phase. Apparently the X-ray adsorption of the kaolin slurry was much stronger than the activated sludge, mainly being attributed to the greater solid contents of kaolin slurries. Multiple tests were required to enhance the resolution of the activated sludge tests. Moreover, flocculation led to rather nonuniform distribution over the cross-section of the sludge sediment, as evidenced by Fig. 2c.

\section{Results and discussion}

\subsection{Time evolutions}

Figs. 3 and 4 depict the transient settling behavior of kaolin slurries and activated sludges, respectively. A supernatant and a constant-solid fraction regime were noted in the first $1000 \mathrm{~s}$ settling of original kaolin slurry
(Fig. 3a). Sediment compaction became apparent after $3000 \mathrm{~s}$ of settling. The highest solid fraction at the bottom was $21 \%$, 21 times higher than the initial solid fraction (ca. 1\%). Flocculation accelerated the settling while sediment compaction started within $100 \mathrm{~s}$ of settling (Fig. $3 b$ for $2 \mathrm{~g} / \mathrm{kg}$ DS and Fig. $3 \mathrm{c}$ for $4 \mathrm{~g} / \mathrm{kg}$ DS). All curves with time greater than $1000 \mathrm{~s}$ coincided with each other, indicating that the compaction had completed. Meanwhile, the flocculant reduced the compressibility of the sludge cake, accompanied with which the maximum achievable solid fraction at tube bottom decreased to about $11 \%$.

For original kaolin slurry test a zone-settling regime at $\phi=\phi_{0}$ was evident at $t<1000 \mathrm{~s}$ of settling. However, we could not identify the constant- $\phi_{\mathrm{g}}$ regime as proposed by the purely elastic model [11]. No such identification was possible for the flocculated slurries owing to their extremely fast settling.

The original activated sludge settled slower than the kaolin slurry (Fig. 4a). Many flocs suspended in the upper liquid layer that made supernatant-slurry inter-

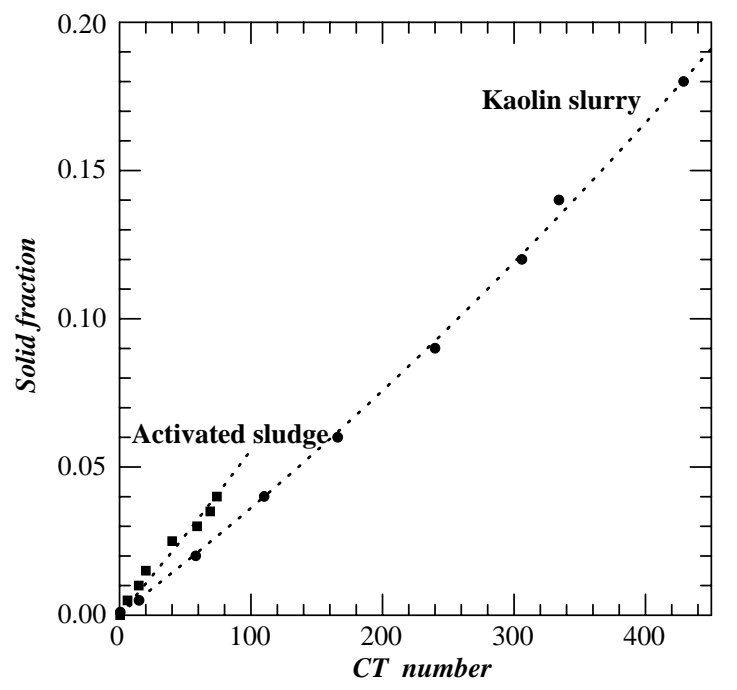

Fig. 1. Solid fraction (v/v) versus the CT number.

Table 1

The $\zeta$-potentials and floc sizes of the original and flocculated sludges

\begin{tabular}{lllllll}
\hline Kaolin slurries $\left(\phi_{0}=1 \%\right)$ & & \multicolumn{3}{l}{ Waste activated sludge $\left(\phi_{0}=0.8 \%\right)$} \\
\cline { 1 - 2 } Dose $(\mathrm{g} / \mathrm{kg}$ DS $)$ & $\zeta$ potential $(\mathrm{mV})$ & Floc size $(\mu \mathrm{m})$ & & Dose $(\mathrm{g} / \mathrm{kg} \mathrm{DS})$ & $\zeta$ potential $(\mathrm{mV})$ & Floc size $(\mu \mathrm{m})$ \\
\hline 0 & -17.7 & 6.3 & 0 & -16.3 & 86.9 \\
0.8 & -12.1 & 31.5 & 2 & -11.2 & 92.1 \\
2 & -10.8 & 41.5 & 8 & -10.1 & 103 \\
4 & -1.2 & 48.5 & 15 & +1.4 & 202 \\
12 & +5.5 & 118 & 30 & +4.8 & 269 \\
\hline
\end{tabular}




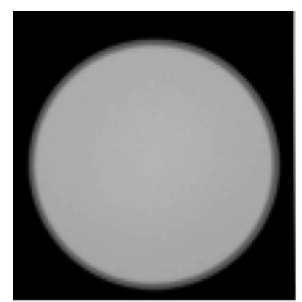

(a)

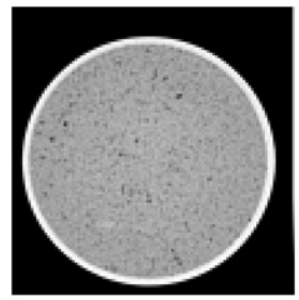

(d)

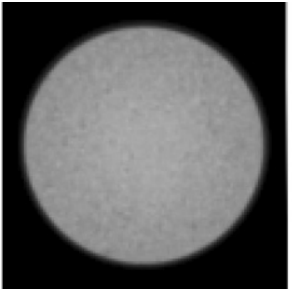

(b)

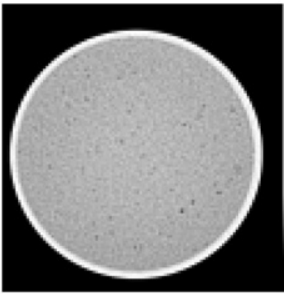

(e)

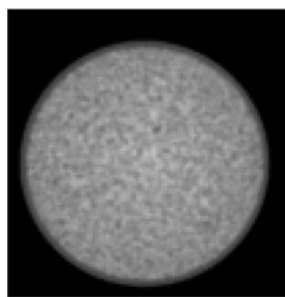

(c)

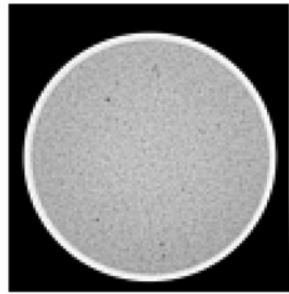

(f)

Fig. 2. The cross-sectional CATSCAN images of the sludge sediment $(0.5 \mathrm{~cm}$ from bottom): (a) original kaolin slurry, (b) kaolin slurry, $2.0 \mathrm{~g} / \mathrm{kg}$ DS, (c) kaolin slurry, $12 \mathrm{~g} / \mathrm{kg}$ DS, (d) original activated sludge, (e) activated sludge, $8 \mathrm{~g} / \mathrm{kg} \mathrm{DS}$, (f) activated sludge, $15 \mathrm{~g} /$ $\mathrm{kg}$ DS.
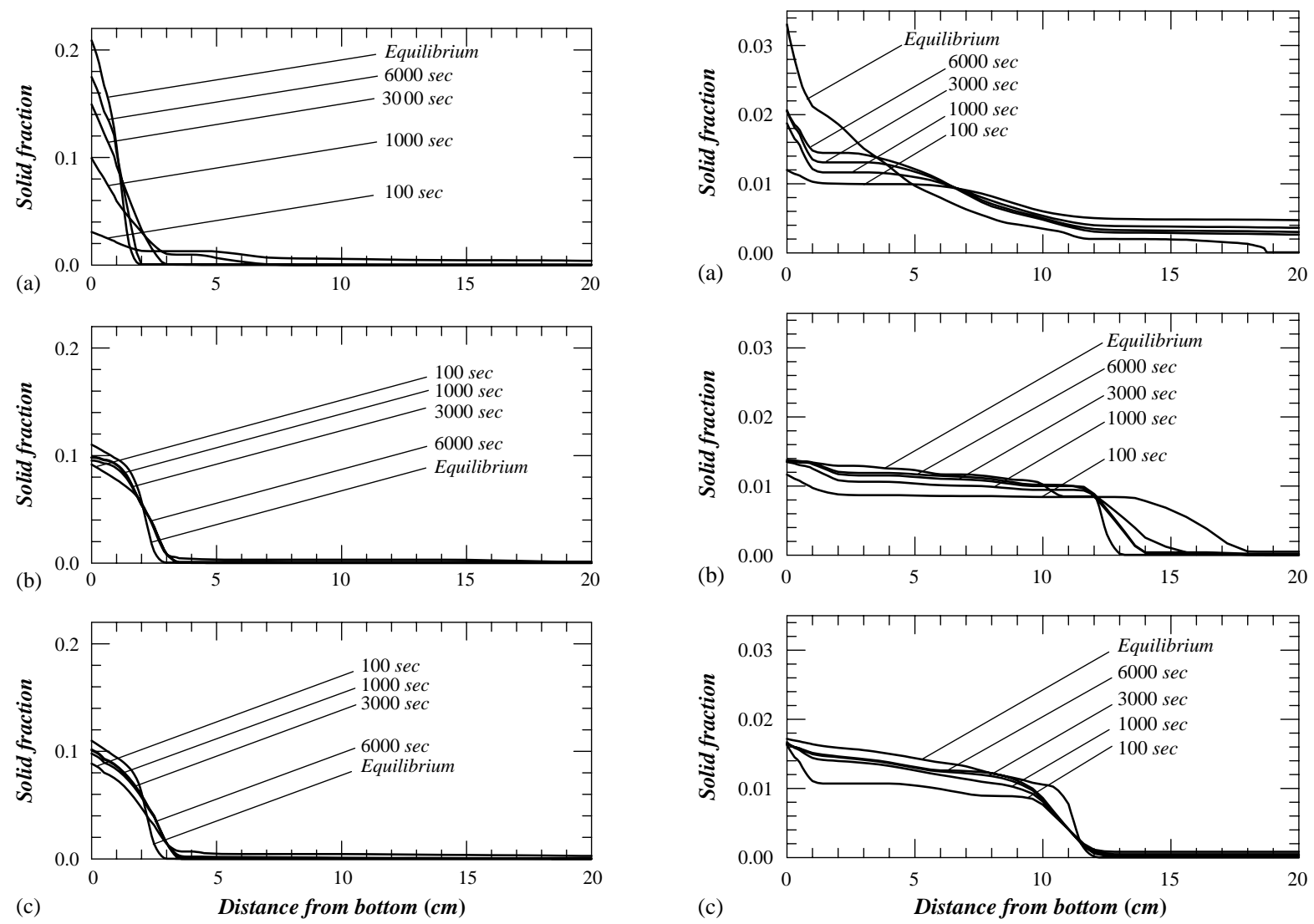

Fig. 3. Transient settling of original and flocculated kaolin slurries: (a) original, (b) $2 \mathrm{~g} / \mathrm{kg}$ DS, and (c) $4 \mathrm{~g} / \mathrm{kg}$ DS.

Fig. 4. Transient settling of original and flocculated waste activated sludge: (a) original, (b) $8 \mathrm{~g} / \mathrm{kg}$ DS, and (c) $15 \mathrm{~g} / \mathrm{kg}$ DS. 
face blurred in the first 6000 s of settling. After 24-hours settling the maximum solid fraction at tube bottom was $3.3 \%$, about 3.1 times higher than the initial fraction (ca. $0.8 \%$ ). Flocculation yielded a satisfactory settleability for the activated sludge (Fig. $4 \mathrm{~b}$ for $8 \mathrm{~g} / \mathrm{kg}$ DS and Fig. 4c for $15 \mathrm{~g} / \mathrm{kg} \mathrm{DS}$ ). (Activated sludge at $2 \mathrm{~g} / \mathrm{kg}$ DS exhibited a poor settling resembling to the original case, whose CATSCAN data are not shown here for brevity sake.) The supernatant was clear and a distinctive supernatant-sediment interface was noticeable over all testing time. Resembling to the kaolin tests, most of the settling process of flocculated activated sludge was in sediment compaction stage rather than in the commonly recognized zone-settling regime.

For the original activated sludge a nearly constant- $\phi$ regime persisted during settling, but with the corresponding $\phi$ values changing with time. For instance, at $t=100 \mathrm{~s}$, the constant $\phi$ was $0.95 \%$ while at $6000 \mathrm{~s}$ the solid fraction became $1.4 \%$. This observation indicates a continuous structural change for the networked flocs during settling. On the other hand, flocculation significantly stiffened the network structure of the waste activated sludge whose constant $\phi$ regime was of a solid fraction around $\phi_{\mathrm{g}}$ (ca. $1.2 \%$ ).

\subsection{Final sediment}

Fig. 5a and b display the spatial distributions of solid fractions in the final sediments of the kaolin slurries and of the activated sludges, respectively. For kaolin slurries, the sediment height increased with the flocculant dose
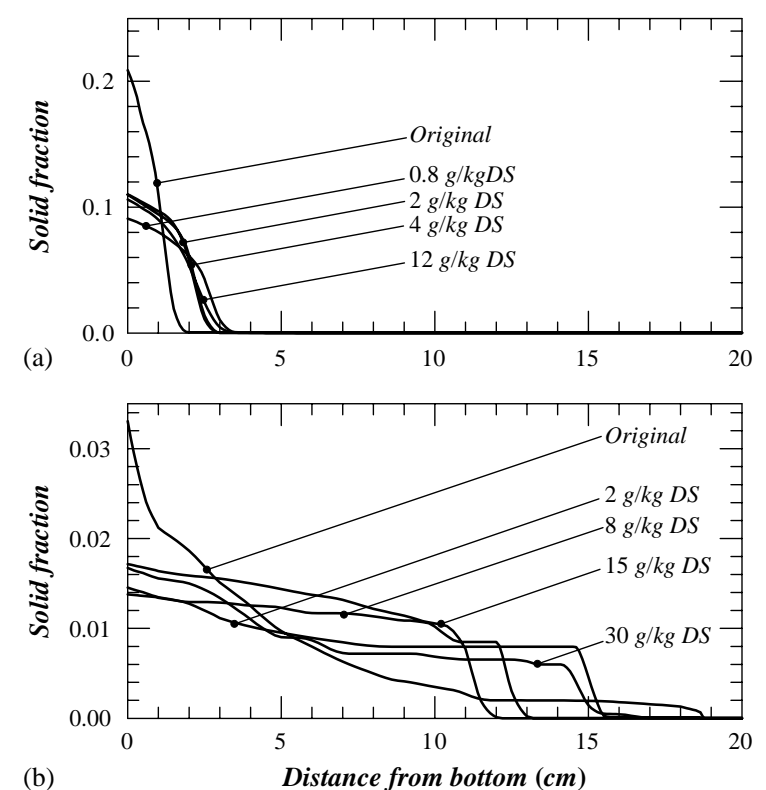

Fig. 5. Solid fraction distributions in the final sediments: (a) Kaolin slurries, (b) waste activated sludges. and reached a maximum at $0.8 \mathrm{~g} / \mathrm{kg}$ DS. Restated, the sludge became looser as more flocculant was added. A loose cake is beneficial for sludge dewatering. The solids fraction distributions of kaolin slurries flocculated at different polymer dosages resembled each other, revealing that intensive flocculation did not affect the packing characteristics of the kaolin particles.

The original activated sludge exhibited a continuous increase in solid fraction from the top surface of the sediment. A rather low solid fraction regime (ca. $0.2 \%$ ) persisted above the underneath sediment after 24-hours settling. Visual observation revealed that the suspended flocs in the upper layer solution should have accounted for the noted low solid fraction regime in Fig. 5b. The solid fraction rapidly increased at $h<11.5 \mathrm{~cm}$, denoting the surface of the sediment. Large pores existed in the final sediment (as shown in Fig. 2c) indicating the occurrence of large water channels during settling [30,31]. This occurrence increased the heterogeneity of the internal structure of the sediment.

For the flocculated samples, on the other hand, the sweep of fine particles by flocculated flocs produced clear supernatant and well-compacted sediment. The reproducibility of the CATSCAN measurements was satisfactory. The solid fraction in the final sediment was less than the original one. Similar to the kaolin slurry tests, flocculation led to bulky sediment that contained more interstitial (intra- or inter-floc) water in sediment.

Although visual observation revealed a clear supernatant-sediment interface for the settling samples, the transition in solids fraction measured using CATSCAN between the sediment and the supernatant was gradual and exhibited no sudden jump in solid fraction over the interface as proposed by most highly idealized theories. A procedure was adopted herein to estimate the $\phi_{\mathrm{g}}$ value, as schematically depicted in Fig. 6. Since the ultimate error for solid fraction estimation was $0.1 \%$, the position where $\phi=0.1 \%$ was taken as the upper surface of the equilibrium sediment. The "interfacial zone" between the suspension and the sediment was taken from the surface $(\phi=0.1 \%)$ to $1 \mathrm{~cm}$ below. The average solid fraction over this $1-\mathrm{cm}$ zone was defined as $\phi_{\mathrm{g}}$, and the results were listed in Table 2. The $\phi_{\mathrm{g}}$ of waste activated sludge kept at around $1.2 \%$. That is, the large and porous activated sludge flocs (with a size of $87 \mu \mathrm{m}$ for original sludge and $200 \mu \mathrm{m}$ for flocculated ones) might entangle with each other, and among these flocs a network formed. Flocculation only mildly influenced the sediment structure of waste activated sludge, as evidenced by the nearly constant $\phi_{\mathrm{g}}$ values in Table 2. For kaolin slurries, on the other hands, $\phi_{\mathrm{g}}$ was $9 \%$ for the original sludge, The value dropped to less than $4 \%$ after flocculation, indicating a loose, networked structure with flocculants. 
The local solid pressure $\left(P_{\mathrm{s}}\right)$ in the final sediment were evaluated as follows:

$P_{\mathrm{s}}=\int_{0}^{z} \frac{\partial P_{\mathrm{s}}}{\partial z} \mathrm{~d} z=\int_{0}^{z}(\Delta \rho g \phi) \mathrm{d} z=\Delta \rho g \int_{0}^{z} \phi \mathrm{d} z$,

where $\Delta \rho$ is the density difference between the solid and the liquid phases, $g$, the gravitational acceleration, and $z$, the vertical distance from the coordinate positioning downward with its origin located at the top of sediment whose corresponding solid fraction is $\phi_{\mathrm{g}}$. The local pressure distribution were evaluated by integrating Eq. (3) with respect of $z$ using data in Fig. 5. Fig. 7 presents the curves for solid fraction versus the calculated solid pressure. Large pores in the flocculated sediment could be identified from the CT images, suggesting that the sediment of kaolin slurry had become looser when the flocculant presented (Fig. 2c). Two linear regimes existed for $\phi$ versus $P_{\mathrm{s}}$ curves on a $\log -\log$ scale for kaolin slurries, dividing each other by a

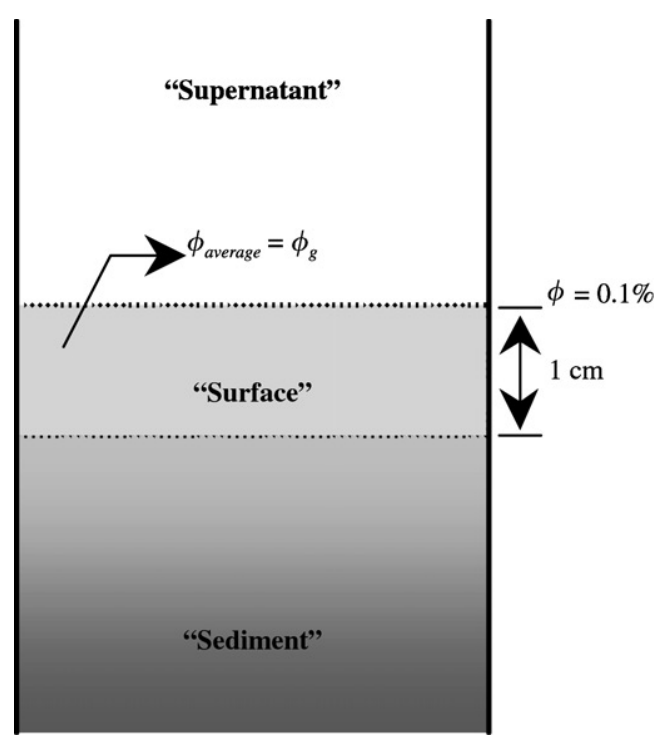

Fig. 6. The schematic description of the $\phi_{\mathrm{g}}$ determination. threshold pressure (approximately 1-2 Pa). Restated, exceeding the threshold pressure the sediment of kaolin slurry was more readily compressible.

The solid fraction of the original activated sludge increased from $1 \%$ to $1.2 \%$ at less than $1 \mathrm{~Pa}$ to more than $3 \%$ at $3-4 \mathrm{~Pa}$. The original activated sludge was much more compressible than kaolin slurries at the low-pressure range investigated. Moreover, flocculation produced bulky sediment (low solid fraction), which was strong enough to resist yielding at applied solid pressure. As Fig. 7 displays, the solid fraction of flocculated sludges only slightly increased from $\phi_{\mathrm{g}}$ to $1.5-2.0 \phi_{\mathrm{g}}$ while the solid pressure exceeded $2 \mathrm{~Pa}$. This observation correlates with the sludge compaction studies [32] and for the centrifugal dewatering of polyelectrolyte flocculated activated sludge [33]. Whether the sediment would further yield over the investigated pressure range $(10 \mathrm{~Pa})$ is not clear.

Eq. (1) correlated the $\phi-P_{\mathrm{s}}$ curves in Fig. 7 using graphical method by Tiller and Leu [10]. The obtained parameters according to the graphical method were adopted as the initial guess for non-linearly regressing the experimental data. Table 2 lists the results. The parameter $P_{0}$ was rather low, generally around or less than $1 \mathrm{~Pa}$, indicating that these slurries would largely deform at the low-pressure regime. Also, the $\beta$ values were higher for waste activated sludge than those for the kaolin slurries $(0.2-0.3$ by Chu and Lee [34] and $\mathrm{Chu}$ et al. [35]), stating that the latter was more compressible than the former. Finally, the higher flocculant dose would yield the lower $P_{0}$ and $\beta$. The sludges after flocculation would deform quickly at the low solid pressure (low $P_{0}$ ) but could resist the external applied pressure at a greater solid pressure (low $\beta$ ). Flocculated sludge would pack more readily when it initially formed. But the sediment became rather "stiff" after initial compaction.

\subsection{Rheological properties}

To analyze the rheological properties of the two sludges, we adopted the model proposed by Landman

Table 2

Model parameters obtained for rheological properties of waste activated sludge

\begin{tabular}{|c|c|c|c|c|c|}
\hline \multicolumn{6}{|c|}{ Waste activated sludge $\left(\phi_{0}=0.8 \%\right)$} \\
\hline Dose (g/kg DS) & $\phi_{\mathrm{g}}(\%)$ & $U_{0} K\left(10^{-14} \mathrm{~m} / \mathrm{s}\right)$ & $P_{\mathrm{y}}(\mathrm{Pa})$ & $P_{0}(\mathrm{~Pa})$ & $\beta$ \\
\hline 0 & 1.2 & ca. 0 & 9.8 & 3.9 & 1.1 \\
\hline 2 & 1.3 & ca. 0 & 9.8 & 2.5 & 0.23 \\
\hline 8 & 1.2 & 1.5 & 5.7 & 5.8 & 0.24 \\
\hline 15 & 1.2 & 1.5 & 3.4 & 1.5 & 0.20 \\
\hline 30 & 1.1 & 1.5 & 5.0 & 1.2 & 0.23 \\
\hline
\end{tabular}




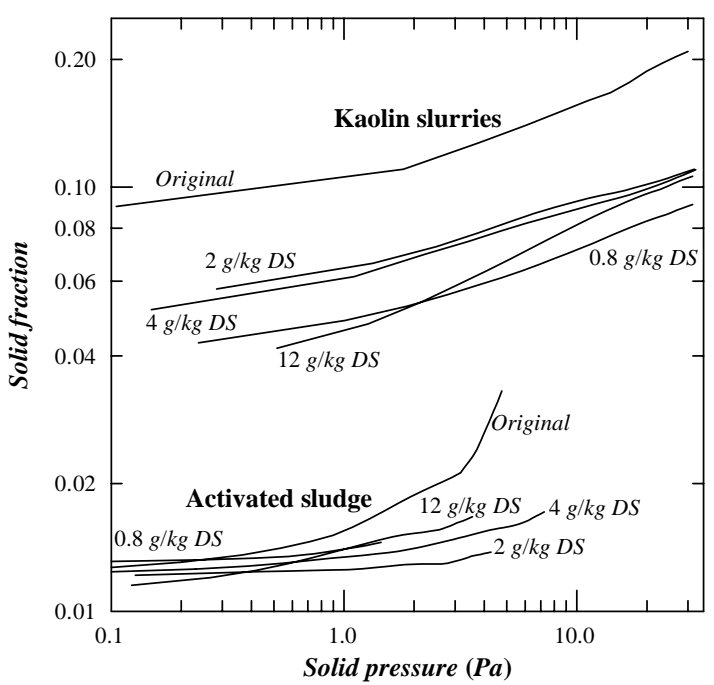

Fig. 7. The solid fraction versus solid pressure plot in the final sediment.

and Russel [36] as follows:

$$
\begin{aligned}
u_{\mathrm{s}}\left(\phi_{0}\right) & =-\frac{\mathrm{d} y}{\mathrm{~d} t}=-U_{0} K\left(\phi_{0}\right)\left(1-\frac{\partial P_{\mathrm{s}} / \partial z}{\Delta \rho g \phi_{0}}\right) \\
& =-U_{0} K\left(\phi_{0}\right)\left[1-\frac{P_{\mathrm{y}}\left(\phi_{0}\right)}{\Delta \rho g \phi_{0}\left(y-L_{\mathrm{c}}\right)}\right],
\end{aligned}
$$

where $U_{0}$ is the terminal velocity of individual particle; $K$ the hindered factor; $y$ the upper interfacial height where $\phi=\phi_{0}$; and $L_{\mathrm{c}}$, the height where $\phi$ deviates from $\phi_{0}$ [15]. A regime of constant $\phi_{0}$ was assumed to exist in the consolidating sediment over the range of $z=y$ where $P_{\mathrm{s}}=0$ to $z=L_{\mathrm{c}}$ where $P_{\mathrm{s}}=P_{\mathrm{y}}$. Hence, the plot with $\mathrm{d} y / \mathrm{d} t$ versus $1 /\left(y-L_{\mathrm{c}}\right)$ estimated the $U_{0} K$ and $P_{\mathrm{y}}$ at $\phi=$ $\phi_{0}$ with linear regression analysis. Eq. (4) was valid only for the consolidating sediment with $\phi>\phi_{\mathrm{g}}$. Apparently the constant- $\phi_{0}$ regime for kaolin slurries was at a solid fraction less than $\phi_{\mathrm{g}}(9 \%$ for original and $4 \%$ for flocculated ones). Only the cases for waste activated sludge warrant further discussion.

Here we took the corresponding height of $\phi=0.5 \phi_{0}$ as $y$ and of $\phi=\phi_{\mathrm{g}}$ as $L_{\mathrm{c}}$ for the calculation. Fig. 8 displays some $\mathrm{d} y / \mathrm{d} t$ versus $1 /\left(y-L_{\mathrm{c}}\right)$ data for the waste activated sludges in the first $500 \mathrm{~s}$. Table 2 lists these obtained $P_{\mathrm{y}}$ and $U_{0} K$ according to regression analysis of Eq. (4). For the original waste activated sludge and that dosed at $2 \mathrm{~g} / \mathrm{kg}$ DS, their supernatants were blurred and the determination of the $y$ values being difficult. Since their settling was rather slow the corresponding $U_{0} K \mathrm{~s}$ were close to zero. Furthermore the $P_{\mathrm{y}}$ 's should be the net weight of the solid phase since under the previous assumption the whole slurry should neither settle nor compact significantly in the early stage of setting. The $P_{\mathrm{y}}$ 's for flocculated sludges over $8 \mathrm{~g} / \mathrm{kg}$ DS largely

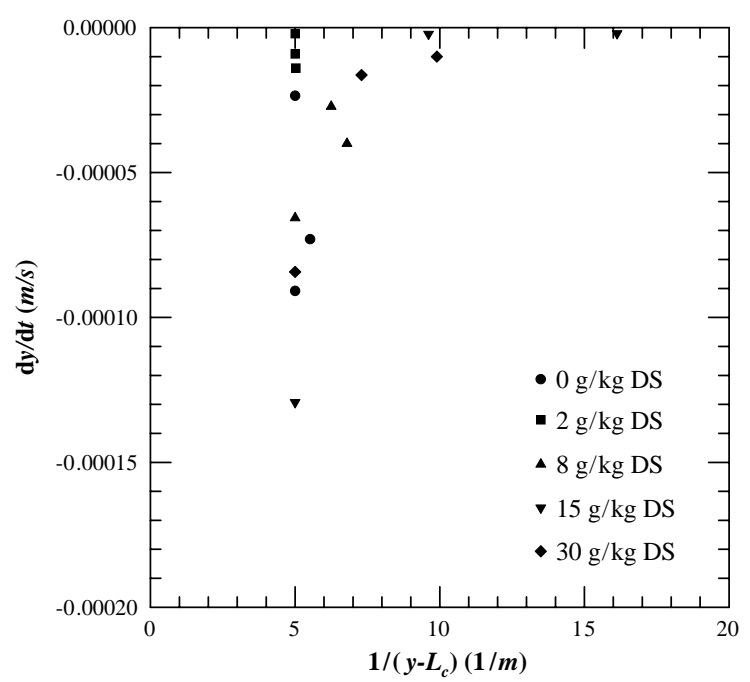

Fig. 8. Plot of $\mathrm{d} y / \mathrm{d} t$ vs. $1 /\left(y-L_{\mathrm{c}}\right)$ of the waste activated sludge during transient settling.

declined to around $5 \mathrm{~Pa}$, with markedly increased $U_{0} K$. Hence, during dynamic settling the flocculation led to the more readily deformed sediment compared with the original sample. A rather low $P_{\mathrm{y}}$ for the waste activated sludge $(<10 \mathrm{~Pa})$ indicated that the solids fraction could be easily enhanced (at least) to its gel point $\left(\phi_{\mathrm{g}}\right)$ regardless of the original sludge concentrations. Restated, in the final clarifier, the waste activated sludge could be easily concentrated to $\phi_{\mathrm{g}}(1.2 \%$ in the investigated case) with the sediment height greater than about $1 \mathrm{~m}$ if the effective floc density $(\Delta \rho)$ were taken as $1 \mathrm{~kg} / \mathrm{m}^{3}$. Flocculated samples are more easily to consolidate than the original sludge.

The $P_{\mathrm{y}}$ values possessed a negative correlation with the $\beta$ 's based on the solid fraction distribution in the final sediment. This occurrence indicates that although the flocculated sludge could be compacted more readily than the original one, the final sediment for the former was stiffer than the latter. Hence, to increase the sediment height could not sufficiently increase the solid fraction in the underflow. The benefit of employing the "superthickener" by Johnson et al. [14] is limited for the waste activated sludge subjected to polyelectrolyte flocculation, which is a common practice in wastewater treatment plant. The data reported herein were beneficial to the theories development for clarifier design and operation.

\section{Conclusions}

This paper reported, for the first time, the spatiotemporal gravitational settling characteristics of activated sludges using CATSCAN. The tests with kaolin 
slurries were conducted for comparison. The settling velocity for the waste activated sludge was much lower than the kaolin slurries. Flocculation markedly enhanced the settleability of activated sludge, but did not markedly reduce its maximum achievable solid fraction. Since the sediment-supernatant interface was blurred for the activated sludge, an averaging procedure estimated the gel-point solid fractions as ca. $1.2 \%$ for the original and the flocculated activated sludge. The solid fraction of the original activated sludge after 24-hours settling increased from $1 \%$ to $1.2 \%$ at less than $1 \mathrm{~Pa}$ to more than $3 \%$ at $3-4 \mathrm{~Pa}$. Further yielding needed higher applied pressure. Flocculation produced bulky sediment with strength to the applied solid pressure. The graphical method by Tiller and Leu [10] correlated the solid fraction distribution in the final sediment. The parameter $P_{0}$ was rather low, generally around or less than $1 \mathrm{~Pa}$, indicating that these slurries would largely deform at the low-pressure regime. The final clarifier could hence easily concentrate the investigated waste activated sludge to $\phi_{\mathrm{g}}(1.2 \%$ in the investigated case). The transient settling for the investigated activated sludges was described using the plastic rheological model by Buscall and White [11]. Flocculation would produce a lower yield stress. Therefore, although the flocculated sludge was tougher than the original sample in the final sediment, the latter was more compressible than the former in the transient settling stage.

\section{Acknowledgements}

National Science Council, ROC, financially supported this work. The assistance from Prof. F.M. Tiller, Prof. K.K. Mohanty, and Dr. Wenping Li of University of Houston on CATSCAN experiments is highly appreciated.

\section{References}

[1] Coe HS, Clevenger GH. Methods for determining the capacities of slime-settling tanks. Trans A I Min Eng 1916;55:356-84.

[2] Kynch GJ. A theory of sedimentation. Trans Faraday Soc 1952;44:166-76.

[3] Talmadge WP, Fitch EB. Determining thickener unit areas. Ind Eng Chem 1955;47:38-41.

[4] Dixon DC. Compression effects in batch settling tests. J Environ Eng ASCE 1982;108:1171-91.

[5] Tiller FM. Revision of Kynch sedimentation theory. AIChE J 1981;27:823-9.

[6] Fitch B. Kynch theory and compression zone. AIChE J 1983;29:940-7.

[7] Font R. Compression zone effect in batch sedimentation. AIChE J 1988;34:229-38.

[8] Font R. Analysis of the batch sedimentation test. Chem Eng Sci 1991;46:2473-82.
[9] Koenders MA, Wakeman RJ. Initial stages of compact formation from suspensions by filtration. Chem Eng Sci 1996;51:3897.

[10] Tiller FM, Leu WF. Basic data fitting in filtration. J Chin Inst Chem Eng 1980;11:61-70.

[11] Buscall R, White LR. The consolidation of concentrated suspensions. Part I: the theory of sedimentation. J Chem Soc Faraday Trans 1987;1(83):873-91.

[12] Auzerais FM, Jackson R, Russel WB, Murphy WF. The transient settling of stable and flocculated dispersions. J Fluid Mech 1990;221:613-39.

[13] Bergstrom L. Sedimentation of flocculated aluminum suspension - gamma-ray measurements and comparison with model predictions. J Chem Soc Faraday Trans 1992;88:3201-11.

[14] Johnson SB, Scales PJ, Dixon DR, Pascoe M. Use of a superthickener device to concentrate potable water sludge. Water Res 2000;34:288-94.

[15] Shen C, Russel WB, Auzerais FM. Colloidal gel filtration: experiment and theory. AIChE J 1994;40:1876-91.

[16] Shin BS, Dick RI. Applicability of Kynch theory to flocculant suspension. J Environ Eng ASCE 1980;106: 505-26.

[17] Chang D, Lee T, Jang Y, Kim M, Lee S. Non-colloidal sedimentation compared with Kynch theory. Powder Technol 1997;92:81-7.

[18] Friedmann T, Windhab EJ. Determination of filter cake properties by nuclear magnetic resonance. Sep Sci Technol 1998;33:2221-39.

[19] La Heij EJ, Kerkhof PJAM, Kopinga K, Pel K. Determining porosity profiles during filtration and expression of sewage sludge by NMR imaging. AIChE J 1996; 42:953-9.

[20] Somasudaran P, Huang YB, Gryte CC. CAT Scan characterization of sedimentation and flocs. Powder Technol 1987;53:73-7.

[21] Bierck BR, Dick RI. In situ examination of effects of pressure differential on compressible cake filtration. Water Sci Technol 1990;22:125-34.

[22] Bierck BR, Dick RI. Mechanisms of compressible sludge cake shrinkage. J Environ Eng ASCE 1990; 116:663-82.

[23] Bierck BR, Wells SA, Dick RI. Compressible cake filtration: monitoring cake formation and shrinkage using synchrotron X-rays. J Water Pollut Control Fed 1988;60:645-50.

[24] David KE, Russel WB, Glantschnig WJ. Disorder-toorder transition in settling suspension of colloidal silica: Xray measurements. Science 1989;245:507-10.

[25] Tiller FM, Yeh CS. Relative liquid removal in filtration and expression. Sep Filtr 1990;27:123-35.

[26] Tiller FM, Hsung NB, Cong DZ. Role of porosity in filtration XII: filtration with sedimentation. AIChE J 1995;41:1153-64.

[27] Buscall R. The sedimentation of concentrated colloidal suspensions. Colloid Surf 1990;43:33-53.

[28] Chang IL, Chu CP, Lee DJ. Filtration followed by expression characteristics of polymer flocculated clay sludge. J Colloid Interface Sci 1997;185:335-42.

[29] Hounsfield GN. A method of and apparatus for examination of a body by radiation such as $\mathrm{X}$ - or 
Gamma-radiation. British Patent No. 1,283,915, London, UK, 1972.

[30] Chen GW, Chang IL, Hung WT, Lee DJ. Regimes of zone settling of waste activated sludge. Water Res 1996; 30:1844-51.

[31] Vesilind PA, Jones GN. Channeling in batch thickening. Water Sci Technol 1993;28(1):59-65.

[32] Chu CP, Lee DJ. Expression of polyelectrolyte flocculated sludge. J Chin Inst Chen Eng 2000;31:321-31.

[33] Chu CP, Lee DJ. Experimental analysis of centrifugal dewatering process of polyelectrolyte flocculated waste activated sludge. Water Res 2001;35:2377-84.
[34] Chu CP, Lee DJ. Solids fluxes in consolidating sediments. J Chin Inst Chem Eng 2001;32:547-54.

[35] Chu CP, Ju SP, Lee DJ, Mohanty KK. Batch gravitational sedimentation of slurries. J Colloid Interface Sci 2002; 245(1):178-86.

[36] Landman KA, Russel WB. Filtration at large pressures for strongly flocculated suspensions. Phys Fluids A 1993; 5:550.

[37] Landman KA, Buscall R, White LR. The continuous flow gravity thickener: steady state behavior. AIChE J 1988; 34:239-52. 